IP Periodica Polytechnica Electrical Engineering and Computer Science

60(1), pp. 51-59, 2016

DOI: $10.3311 /$ PPee.9046

Creative Commons Attribution (i)

RESEARCH ARTICLE

\section{Design of Small Printed Multiband Loop Antennas for Short Range Wireless (SRW) Applications}

\author{
Attila Zólomy ${ }^{1 *}$, Tamás Bódi ${ }^{1}$, Eric Unruh $^{2}, Z$ Zoltán Vida ${ }^{1}$
}

Received 02 October 2015; accepted 30 December 2015

\begin{abstract}
Worldwide application of wireless IoT and telemetry solutions are more cost effective (require lower manufacturing and logistic cost) and more immune against interferences, if they are inherently able to operate in multiple frequency bands. Switched current high impedance PA configurations driving high impedance printed antennas with very few discrete components have very low cost and yields good efficiency in the $0 \ldots+10 \mathrm{dBm}$ power range. However, one major bottleneck is the difficulty of the high impedance multiband antenna design.

In this paper, investigation, design and measured results of multiband, high impedance, small, printed UHF loop antennas are presented. The built antenna are inherently tuned with only two discrete capacitors to properly terminate the applied high impedance, highly efficient switched current power amplifier (PA) in the targeted two UHF bands. The presented design method is applicable for more than two band operations and for higher frequency bands as well.
\end{abstract}

\section{Keywords}

IoT devices, Multiband antenna design, inherently tuned small loop, low bill of material, high impedance efficient PA

\footnotetext{
${ }^{1}$ Silicon Laboratories Hungary Kft.,

H-1037 Budapest, Záhony u. 7, Hungary

${ }^{2}+$ Silicon Laboratories Sunnyvale office

940 Stewart Drive, Sunnyvale, CA 94085, USA

*Corresponding author, e-mail: zolomy@silabs.com
}

\section{Introduction}

Cost reduction is a major driving force in short range wireless (SRW) application design. Rapid spreading of door openers, panic buttons for elderly/disabled peoples, intelligent home systems, automatic meter reading (AMR), mesh networks and other internet of things (IoT) solutions (smart watches, bulbs, sensors, phones etc.) can be observed in the recent years due to this. Beside the low manufacturing cost (e.g. crystal less, cheap radio ICs with printed antennas and few discrete components), low worldwide logistics and maintenance cost (e.g. long battery life) are essential as well. Radios able to operate at several bands (allowed by the different regional standards) can be manufactured and spread-out worldwide in higher volumes with lower expenses.

Increasing number of radio devices also makes the interference problems more critical. A typical example is the EU 868M ISM band where the 4G LTE mobile technology causes serious interference problems. A radio link with multiband capability can vary the operational band if strong link degradation occurs due to the interferences.

Design of multiband printed antennas are challenging. Popular multiband antenna types are the unbalanced, low impedance (50 Ohm) monopoles and its derivatives like PIFA or IFA [1]. However, in the $0 \ldots+10 \mathrm{dBm}$ power regime higher impedance antennas (driven by high impedance PAs) can work more efficiently in the typical $2 \ldots 3.6 \mathrm{~V}$ supply voltage range [2]. Other configurations with good efficiency applies either switching mode (Class E, Class C etc.) PAs [3], or a 50 Ohm system with a DCDC converter. However, both has disadvantages. One of them is the increased bill of material and cost. Switching mode PAs generate rather strong harmonics and thus, need complicated matching with strong filtering. A DCDC converter solution is inherently expensive and besides, needs a complicated and expensive supply filtering to avoid up converted spurs (i.e. the so-called "Christmas three" spectrum). Other problem with the switching mode PAs is the high voltage peak on the output FET's drain, which arise reliability issues.

One popular low cost, high impedance antenna type is the small loop [4]. It is inherently inductive, so it can be tuned at 
its input by a simple parallel capacitance, which can be a simple discrete capacitor and/or the radio driver's output parasitic capacitance. Use of capacitors as matching elements are desirable as they are cheaper than inductors and have significantly higher Q, resulting in lower loss. Application of high Q matching elements is especially important in case of loop antennas as practical small size loops have very high Q ( $\sim$ several hundred) and thus, high parallel equivalent resistance $(\sim 20 \ldots 40 \mathrm{KOhm})$. Matching of such a very high impedance is challenging due to the losses and element spreading sensitivity. One method to handling this is the so-called "tapped loop" technique, which can transform the loop impedance down to several hundred Ohms. This impedance level is a usable termination for PAs working in the $0 \ldots+10 \mathrm{dBm}$ power range and from $2 \ldots 3.6 \mathrm{~V}$ supply voltage. Other advantage of the tapping is that together with the impedance the inductance shown at the antenna input is decreased as well. By this way, a larger main loop can be used with the same input parallel tuning cap (i.e. with the same PA parasitic cap), which improves the radiation significantly.

The tapping can be either inductive or capacitive. Inductive tapped loops comprise two loops with inductive coupling between them. Due to this, the design is difficult and in practice, cannot be done without EM simulators. As opposed to this, there are analytical formulas for the capacitive tapping $[5,6]$ and the design process is much simpler. Capacitive tapped loops apply a series cap in the main loop, which together with the input parallel cap transforms down the antenna impedance. The structure and the equivalent circuit of a capacitive tapped loop is shown in Fig. 1 and Fig. 2, respectively The input parallel capacitor (denoted by Cpar) is usually the PA capacitance of the RF IC. If its value is not high enough a discrete cap can be used together with the PA cap. The input impedance $\left(R_{I N}\right)$ is usually much lower than the loop impedance $\left(R_{P}\right)$ as the $(\mathrm{Cpar} / \mathrm{Cser})^{2}$ ratio is usually very high. However, due to this a slight variation in the series cap (Cser) strongly detunes the antenna and a large change in the value of Cpar is required for the compensation. RF ICs with built in automatic antenna tuning functionality [7] can handle this to some extent.

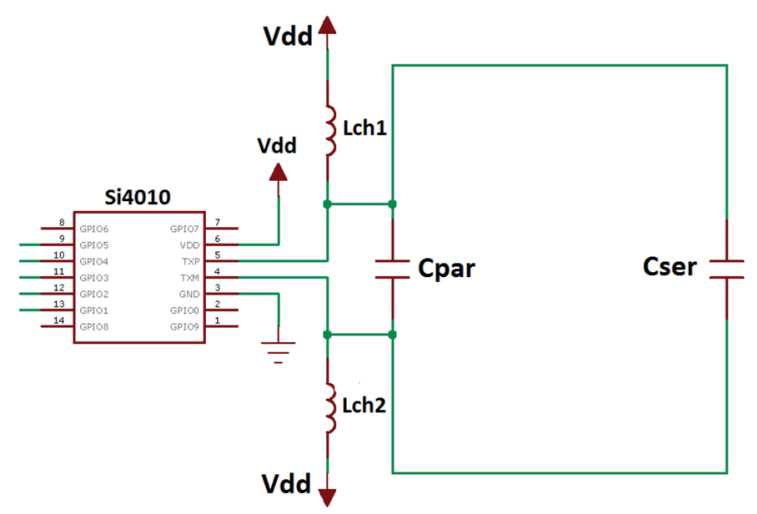

Fig. 1 Typical capacitive tapped loop structure with DC feed inductors (Lch1 and Lch2)

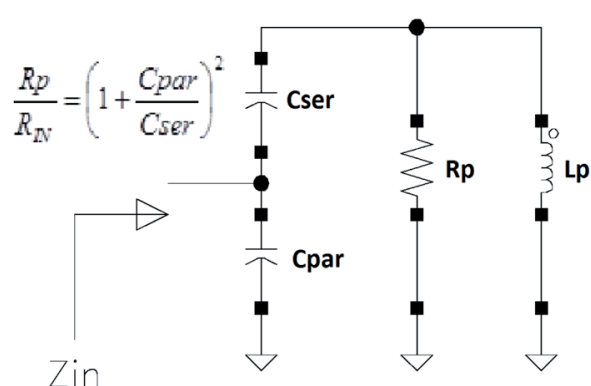

Fig. 2 Simplified equivalent circuit of a typical capacitive tapped loop structure of Fig. 1

The loop antenna can be either a differential or single ended one. Single ended antennas are usually half of the differential counterpart (monopole $<->$ dipole, half loop $<->$ normal loop etc.), but require a large ground plane (perpendicular to the antenna axe/plane, if possible), which behaves as an electrical mirror. From design point of view of multiband antennas, the topology (differential or single ended) is not important; the presented method is proper both for single ended and differential loop antennas.

\section{Multiband Loop Antenna Design}

The basic dual band, capacitive tapped loop antenna contains a second small loop as shown in Fig. 3. The small loop has parallel resonance with the PA capacitance (Cpar) while the main loop has a series resonance with the series capacitance (Cser). The equivalent circuit comprise these two resonators as shown in Fig. 4.

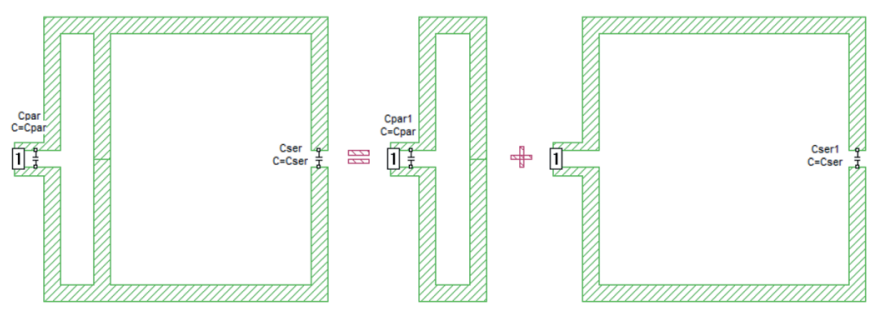

Fig. 3 Capacitive tapped dual band loop decomposition into a small normal loop and a large loop with series discrete capacitor

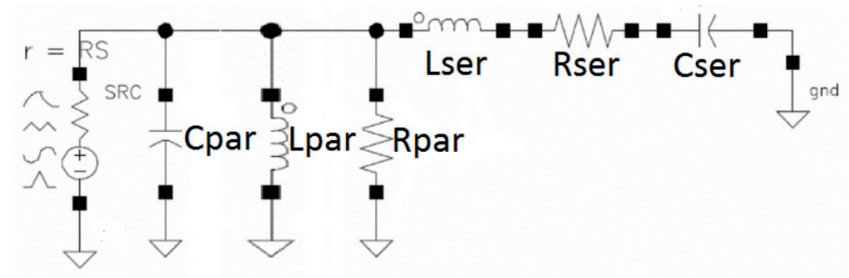

Fig. 4 Equivalent circuit of the capacitive tapped dual band loop.

Assuming identical parallel and series resonant frequencies, the parallel resonating small loop behaves as a shunt tuning inductor below and as a shunt tuning capacitor above the resonant frequency. Fig. 5 and Fig. 6 show the impedance variation on a Smith Chart. In Figure 5 the blue curve shows the series resonating main loop impedance locus without the 
parallel resonating small loop. The two other curves (pink and orange) shows the case when a proper parallel reactance (pink $=$ inductance below resonance, or orange $=$ capacitance above resonance) is introduced. With the addition of the parallel reactances, the curves approach the horizontal axis of the Smith chart in a parallel resonance fashion. If the reactances are simultaneously provided by a real parallel resonant circuit, two parallel resonances show up on both sides of the series resonance as shown in Fig. 6 and Fig. 7 (magnitude curve).

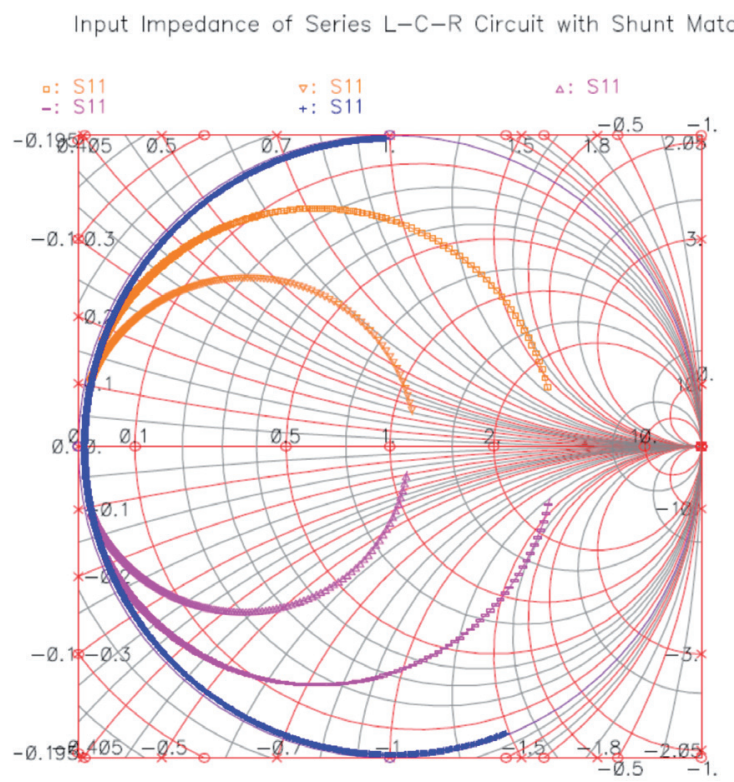

Fig. 5 Impedance locus of the series resonating big loop alone (blue curve), and with parallel reactances (pink curve with inductance, orange curve with capacitance).

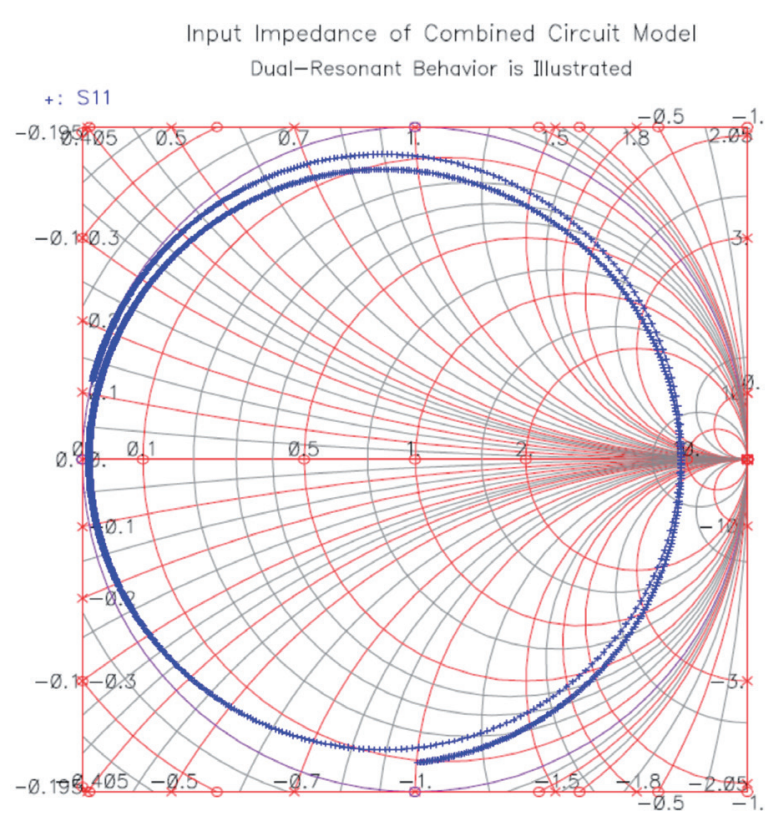

Fig. 6 Impedance locus of the antenna equivalent circuit of Fig. 4, with identical series and parallel resonant frequencies. The interaction between the two resonators yields two parallel resonances below and above the series resonance Magnitude of Input Impedance of Combined Circuit Model
Dual-Resonant Behavior is Illustrated

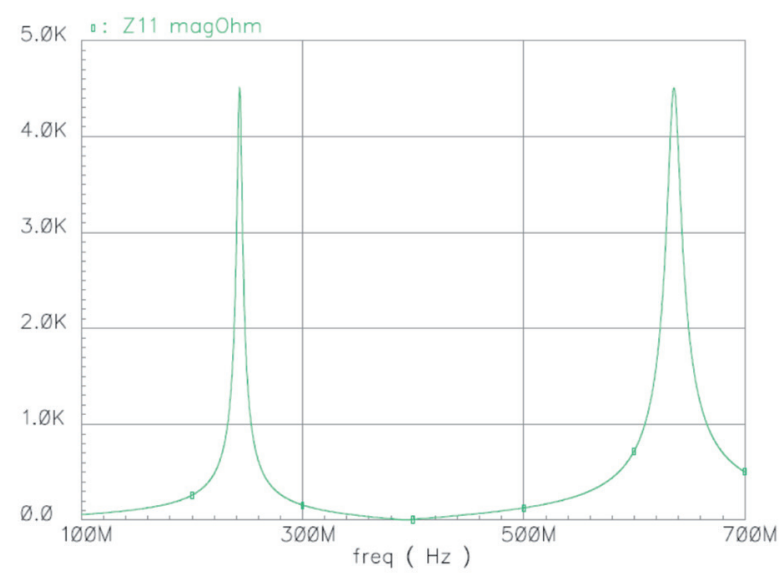

Fig. 7 Impedance magnitude curve of the case of Fig. 6. Parallel resonances appear below and above the series resonance

\section{Analytical Calculation of the Dual Band Loop}

The two parallel resonances and the series resonance of the dual band loop configuration can be analytically calculated using the equivalent circuit of Fig. 4. Equation (1) shows the input admittance:

$$
Y_{I N}=\frac{1}{J \omega L_{P A R}}+J \omega C_{P A R}+\frac{1}{R_{P A R}}+\frac{1}{J \omega L_{S E R}+\frac{1}{J \omega C_{S E R}}+R_{S E R}}
$$

The aim is to calculate the new resonant frequencies, besides both resonators have high $\mathrm{Q}$. So to simplify the calculation the losses are neglected.

$$
Y_{I N}=\frac{1-\omega^{2} C_{P A R} L_{P A R}}{J \omega L_{P A R}}+\frac{J \omega C_{S E R}}{1-\omega^{2} L_{S E R} C_{S E R}}
$$

After some algebraic steps one can derive the pure reactive input admittance.

$$
Y_{I N}=-j \frac{\left(1-\frac{\omega^{2}}{\omega_{0}^{2}}\right)^{2}-\frac{\omega^{2}}{\frac{1}{C_{S E R} L_{P A R}}}}{\omega L_{P A R}\left(1-\frac{\omega^{2}}{\omega_{0}^{2}}\right)}=-j \frac{\left(1-\frac{\omega^{2}}{\omega_{0}^{2}}\right)^{2}-\frac{\omega^{2}}{\omega_{\text {OFFS }}^{2}}}{\omega L_{P A R}\left(1-\frac{\omega^{2}}{\omega_{0}^{2}}\right)}
$$

Where $\omega_{0}$ is the original resonant frequency and $\omega_{\text {OFFS }}$ is a relative offset:

$$
\omega_{0}=\frac{1}{L_{P A R} C_{P A R}}=\frac{1}{L_{S E R} C_{S E R}}, \quad \omega_{O F F S}=\frac{1}{L_{P A R} C_{S E R}},
$$

The denominator of Eq. (3) shows that the series resonance i.e. where the $Y_{I N}$ has a pole, is at the original $\omega_{0}$ resonant frequency. While the parallel resonances (i.e. where the nominator is zero) are detuned. To calculate them the nominator of Eq. (3) should be decomposed into multiplication factors as 
shown in Eq. (5). At the parallel resonances the nominator is zero i.e. the multiplication factors have to be zero.

$$
\left(1-\frac{\omega^{2}}{\omega_{0}^{2}}\right)^{2}-\frac{\omega^{2}}{\omega_{\text {OFFS }}^{2}}=\left(1-\frac{\omega^{2}}{\omega_{0}^{2}}+\frac{\omega}{\omega_{\text {OFFS }}}\right)\left(1-\frac{\omega^{2}}{\omega_{0}^{2}}+\frac{\omega}{\omega_{\text {OFFS }}}\right)
$$

From this condition two second order equations yield for the parallel resonant frequencies (Eq. (6) and (7)). Solving these equations four frequency solutions are derived (Eq. (8) and (9)).

$$
\begin{gathered}
\frac{\omega^{2}}{\omega_{0}^{2}}-\frac{\omega}{\omega_{\text {OFFS }}}-1=0 \\
\frac{\omega^{2}}{\omega_{0}^{2}}+\frac{\omega}{\omega_{\text {OFFS }}}-1=0 \\
\omega_{1,2}=\frac{\frac{1}{\omega_{\text {OFFS }}} \pm \sqrt{\frac{1}{\omega_{O F F S}^{2}}+\frac{4}{\omega_{0}^{2}}}}{\frac{2}{\omega_{0}^{2}}} \\
\omega_{3,4}=\frac{-\frac{1}{\omega_{\text {OFFS }}} \pm \sqrt{\frac{1}{\omega_{O F F S}^{2}}+\frac{4}{\omega_{0}^{2}}}}{\frac{2}{\omega_{0}^{2}}}
\end{gathered}
$$

As $L_{P A R}<<L_{S E R}$ and $C_{S E R}<<C_{P A R}$ then $\omega_{0}<<\omega_{\text {OFFS }}$, several simplifications are possible in Eq. (8) and (9).

$$
\begin{aligned}
\omega_{1,2} \cong & \frac{1}{\omega_{\text {OFFS }}} \pm \frac{2}{\omega_{0}} \\
\frac{\omega_{0}^{2}}{2 \omega_{\text {OFFS }}} \pm \omega_{0} & \\
\omega_{3,4} \cong & \frac{-\frac{1}{\omega_{\text {OFFS }}} \pm \frac{2}{\omega_{0}}}{\frac{2}{\omega_{0}^{2}}}=\frac{-\omega_{0}^{2}}{2 \omega_{\text {OFFS }}} \pm \omega_{0}
\end{aligned}
$$

Again as $\omega_{0}<<\omega_{\text {OFFS }}$, the offset part is much smaller than the $\omega_{0}$ and thus, only two solutions falls to the positive frequency range (Eq. (12) and (13)). They are the real parallel resonant frequencies shifted by the offset part around the original $\omega_{0}$ resonance.

$$
\begin{gathered}
\omega_{p 1}=\omega_{0}+\frac{\omega_{0}^{2}}{2 \omega_{\text {OFFS }}} \\
\omega_{p 2}=\omega_{0}-\frac{\omega_{0}^{2}}{2 \omega_{\text {OFFS }}}
\end{gathered}
$$

\section{Tuning of the Dual band Loop}

The resonances behave differently for the tuning of the parallel or series resonant circuit. Figure 8 shows the case when the Lpar/Cpar ratio is varied (while maintaining a constant
Lpar*Cpar product i.e the parallel resonant frequency is fixed). With increasing the ratio, both the frequency offset (i.e., spacing between the two parallel resonant frequencies) and the resonant impedances increase.

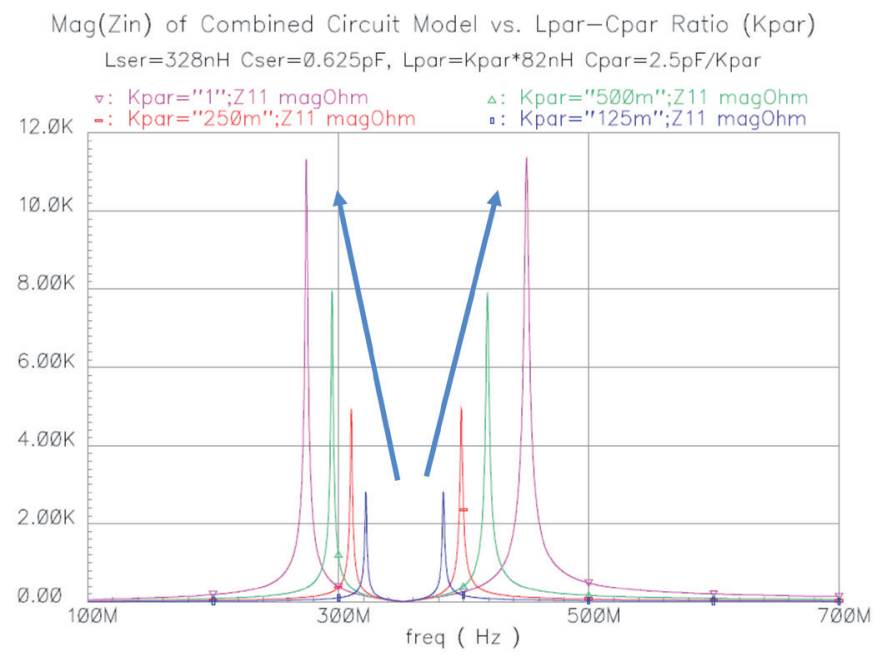

Fig. 8 Impedance magnitude curve change if the Lpar/Cpar ratio is varied (the resonant frequencies and the $L$ ser/Cser ratio are fixed)

Figure 9 shows the effect of the Lser/Cser ratio change (again the $L \mathrm{ser}^{*}$ Cser product i.e. the series resonant frequency is constant). Here with increasing ratio the frequency offset decreases and the impedances increase. The design target is to adjust the resonances to the specific desired operating frequencies, and to simultaneously adjust the impedances of the resonances to the targeted value. The locations of the two parallel resonances can be tuned by the series resonant frequency (which again equals to the parallel one) and by the offset. Many good solutions (i.e. many good Lser/Cser and Lpar/Cpar ratio pairs) exist if the designer is free to choose the impedance. If the impedances are also specified, then only one solution exists as in this case both the $L / C$ ratios and the $L-C$ products (i.e. the resonant frequencies) are fixed.

Some secondary effects also have slight influences. These are the losses and the coupling between the loops. The losses are mainly determined by the capacitor Qs, so can be considered as constants independently from the loop size tuning. The same is assumed for the coupling.

The required impedance value depends on the target power level and on the allowed voltage swing on the PA. The Si4010 type RF IC requires $\sim 600$ Ohm differential termination impedance at $3 \mathrm{~V}$ supply voltage to deliver $\sim+9 \ldots+10 \mathrm{dBm}$ to the antenna at the targeted frequencies [8]. In Figure 10 a dual band ( 315 \& $390 \mathrm{MHz}$ ) loop example design is shown with fixed PA cap $($ Cpar $=28.4 \mathrm{pF})$, and with a $C$ ser value of $4.2 \mathrm{pF}$. The $Q$ of the applied 0402 SMD capacitors are typically around hundred, so the values of the parallel equivalent losses of the caps (denoted by $R p a r \_Q$ and $R s e r \_Q$ ) are approx. $2 \mathrm{~K}$ and $11 \mathrm{~K}$, respectively. The antenna is designed with the Sonnet planar EM simulator [9]. The antenna impedance is the proper $\sim 550 \ldots 600 \mathrm{Ohm}$ at both 
target frequencies as shown in Fig. 11. Unfortunately, the resulted size of the main loop is very small, only $14 \mathrm{~mm}$ by $14 \mathrm{~mm}$, which yields a very poor antenna gain at the targeted operation frequencies: that is $\sim-32 \mathrm{dBi}$ at $315 \mathrm{MHz}$ and $\sim-25 \mathrm{dBi}$ at $390 \mathrm{MHz}$. It can be concluded that the proper solution which yields the proper resonant frequencies with the proper impedances may not have a large enough loop size for good radiation efficiency.

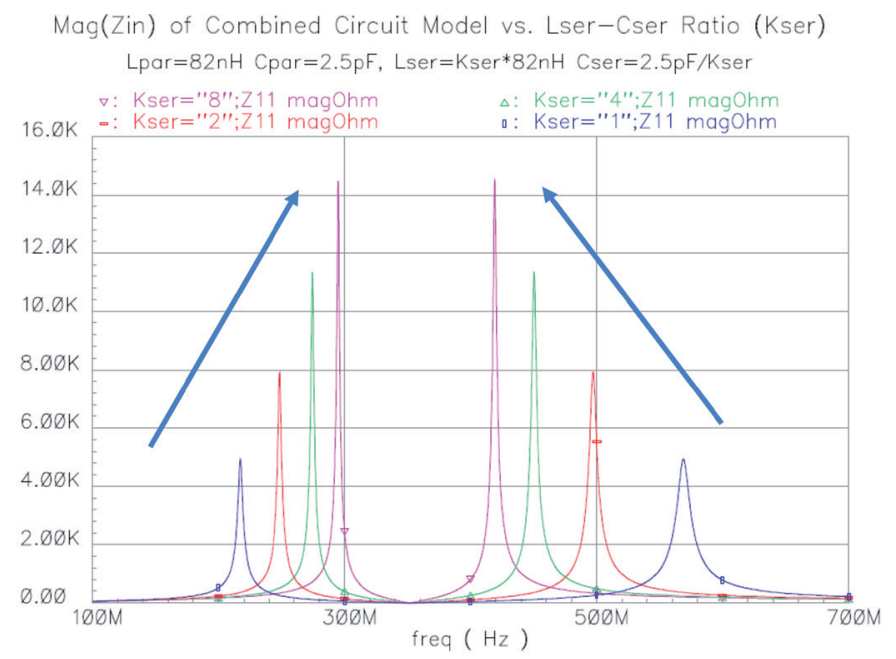

Fig. 9 Impedance magnitude curve change if the Lser/Cser ratio is varied (the resonant frequencies and the Lpar/Cpar ratio are fixed)

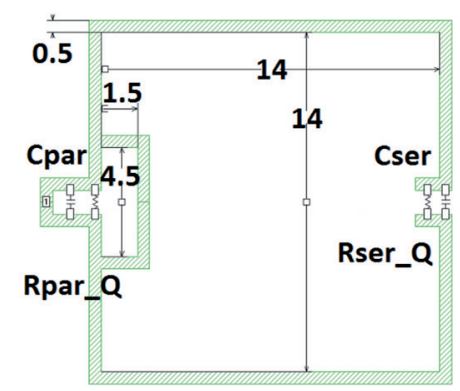

Fig. 10 Dimensions of the designed 315 \& $390 \mathrm{MHz}$ dual band loop

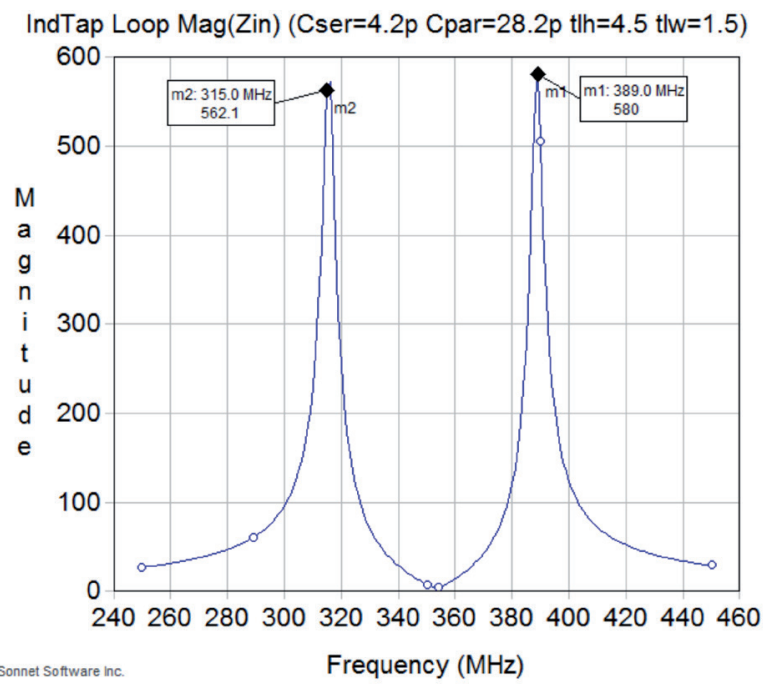

Fig. 11 Impedance magnitude curves of the designed 315 \& $390 \mathrm{MHz}$ dual band loop. The targeted impedance at resonance is $\sim 600 \mathrm{Ohm}$

\section{Dual Band Loop Design with Variable PA Capacitance}

A possible way to overcome the problem is to introduce an additional design of freedom i.e. the variation of the PA capacitance (Cpar). This method will be investigated here. The Si4010 radio chip has a built in automatic antenna tuning circuitry, which tunes the PA output capacitance (i.e. Cpar) to maintain the resonance at the output. This feature is used here. The general equivalent circuit of the multiband loop antenna is shown in Fig. 12.

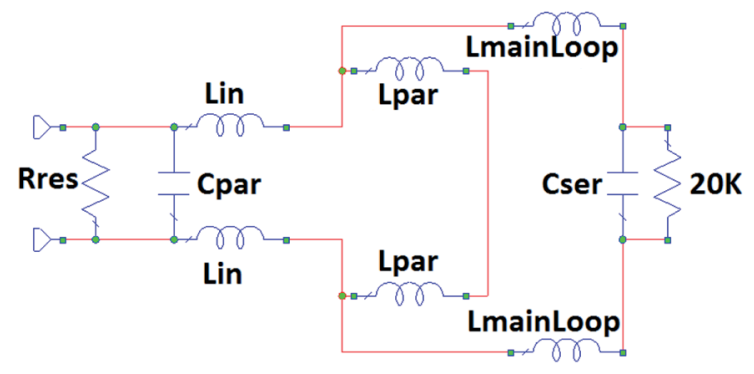

Fig. 12 Equivalent circuit of the dual band loop of Fig. 10

Formulas for the inductance $(L)$ and radiation resistance (which is proportional to the gain) of electrically small rectangular loops are given by Eq. (14) and (15) [4]. The dimension parameters are used in the formulas are shown in Fig. 13.

$$
\begin{gathered}
L=\frac{2 \mu_{0} \sqrt{a_{1} a_{2}}}{\pi}\left[\ln \left(\frac{a_{1} a_{2}}{b}\right)-0.774\right] \\
R_{R A D}=320 \pi^{4}\left[\frac{A^{2}}{\lambda^{4}}\right]=320 \pi^{4}\left[\frac{A^{2} f^{4}}{v^{4}}\right]
\end{gathered}
$$

where:

$A$ is the area of the loop: $A=a 1 * a 2$ and $b$ is an effective cross section derived from the printed trace cross section dimensions: $b=0.35 * t+0.24 * w$.

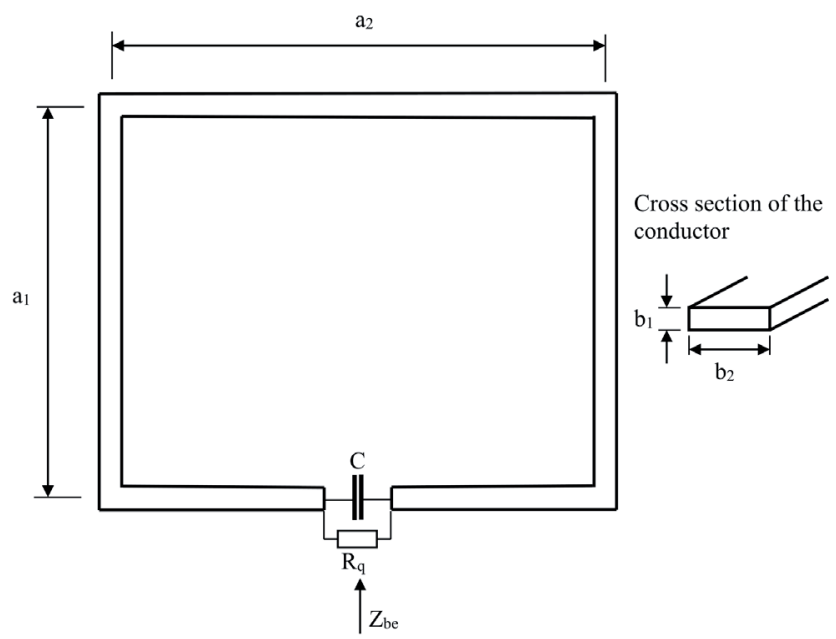

Fig. 13 Loop dimension parameters for gain and inductance calculations 
The $14 \mathrm{~mm}$ by $14 \mathrm{~mm}$ main loop size with $0.5 \mathrm{~mm}$ trace width of the previous antenna design (Fig. 10) yields approx. $\sim 52 \mathrm{nH}$ loop inductance and $\sim-25 \mathrm{dBi}$ antenna gain at 390 $\mathrm{MHz}$ (here the effect of the losses are also taken into account). For better gain significant increase of the main loop size is required, with increased main loop inductance as well. With the larger main loop the design deviates from the optimum dual band solution of Fig. 10, which has the right impedances at the targeted bands with fixed PA capacitance (Cpar).

The main problem with the larger main loop (i.e. with larger Lser/Cser ratio) is that the offset decreases and the impedance peaks increase as shown in Fig. 9. As can be seen in Fig. 8 to reduce the impedance back to $\sim 550 \ldots 600 \mathrm{Ohm}$, a smaller Lpar/Cpar ratio i.e a reduced small loop size is needed (parallel with the small loop reduction the increase of Cpar needed to keep the resonant frequency fixed). Unfortunately, this decreases the offset further, so after these tuning steps the frequency offset is far less than required. In this situation the only possibility to tune the resonances to the targeted operation frequencies is to adjust PA capacitance (Cpar) as well. That is, Cpar should be decreased/increased properly when the radio operates at the highest/lowest targeted frequency. With this method the impedance is optimum only at one band at a given time, but that is not a big disadvantage as usually the short range radios does not transmit at multiple bands simultaneously. Usually, they change the bands in a time division fashion and the PA should change its capacitance accordingly. For these operation an automatic antenna tuning feature like the one applied in the Si4010 RF IC is required.

The simulated impedance magnitude curve of an antenna using this approach is shown in Fig. 14. To achieve the $\sim 550 \ldots 600 \mathrm{Ohm}$ resonant impedances at $315 \mathrm{MHz}$ and 390 $\mathrm{MHz}$, the Cpar must be tuned to $\sim 80 \mathrm{pF}$ and $\sim 61 \mathrm{pF}$, respectively. That is $\sim 19 \mathrm{pF}$ tuning range. The element values of the equivalent circuit for this antenna are shown in the second column of Table 1. The main loop inductance is chosen to be high: it is $2 * 84 \mathrm{nH}$ compared to the $\sim 52 \mathrm{nH}$ of the previous 14 $\mathrm{mm}$ by $14 \mathrm{~mm}$ case. The 3.5 times higher main loop inductance allows a size of $\sim 35$ by $35 \mathrm{~mm}$. With this higher size approx. -14 $\mathrm{dBi}$ antenna gain (incl. losses) can be achieved, which is a significant, $\sim 11 \mathrm{dBi}$ improvement.

However, there are problems with the realization. The first problem is that the required Cpar tuning range is $\sim 19$ $\mathrm{pF}$, which is higher than the available PA capacitance tuning range of the applied $\operatorname{Si4010}(\sim 2.7 \mathrm{pF} \ldots 12.5 \mathrm{pF}=9.8 \mathrm{pF}) \mathrm{RF}$ IC. Moreover, the tuning range of the applied RFIC should be significantly higher than the required by the antenna to compensate other effects, like technological spreading or hand effect. The second problem is the very small inductance value of the small loop ( $2 *$ Lpar). It is only $6 \mathrm{nH}$, which is practically cannot be realized. The third big problem is that the common route inductance of the small and main loop (denoted by Lin in the equivalent circuit) should be zero i.e. it should be a short with zero physical length. Due to these issues this dual band loop design cannot be realized in practice. So the approach of varying solely Cpar is not proper to create a practically realizable high gain dual band solution.

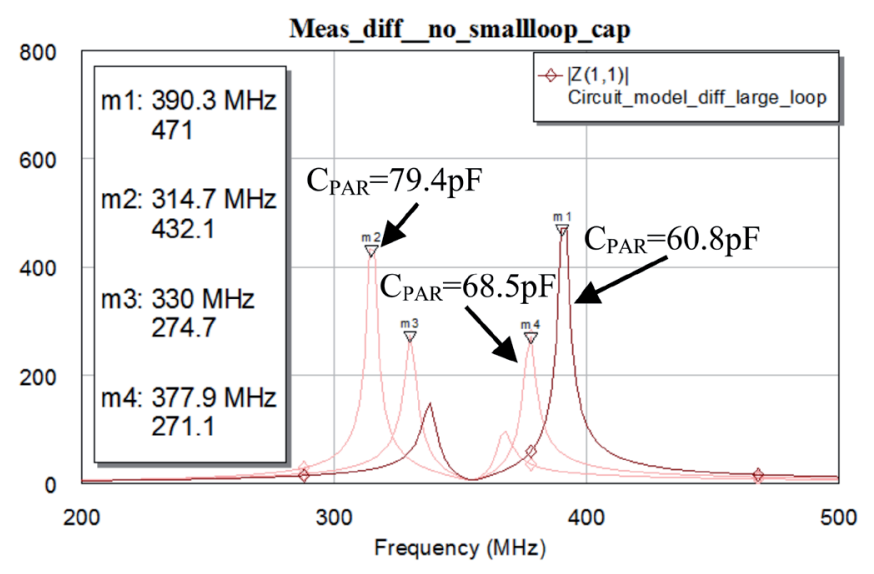

Fig. 14 Simulated impedance curves of the increased main loop case with different Cpar values.

\section{Dual Band Loop Design with Introducing a Second Series Capacitance in the Small Loop}

A possible way to overcome the above mentioned realization problems is to introduce an additional series capacitance (Cser2) in the small loop. Figure 15 shows the equivalent circuit of an antenna like this. The third column of Table 1 shows the equivalent circuit element values of a loop designed in this way. Here the main loop inductance $(\sim 2 * 83 \mathrm{nH})$ is the same as in the previous case, but the small loop inductance can be much higher (Lpar=22 $\mathrm{nH}$ ) as the series connected Cser2 partly compensates its reactance. The higher Lpar value results a practically realizable small loop size.

Table 1

\begin{tabular}{lll}
\hline Rres & $1.3 \mathrm{~K}$ & $2 \mathrm{~K}$ \\
\hline Cpar & $60.8 \ldots 79.4 \mathrm{pF}$ & $13.8 \mathrm{pF}$ \\
Lin & Short & $1 \mathrm{nH}$ \\
Lpar & $3 \mathrm{nH}$ & $11.9 \mathrm{nH}$ \\
Cser2 & short & $22 \mathrm{pF}$ \\
Lmain-Loop & $84 \mathrm{nH}$ & $83 \mathrm{nH}$ \\
Cser & $1.2 \mathrm{pF}$ & $1.2 \mathrm{pF}$ \\
\hline
\end{tabular}

The simulated impedance magnitude vs. frequency curves at different sizes of the small loop are shown in Fig. 16. Here the Cser 2 cap is always tuned to yield a $\sim$ symmetrical two peak shaped impedance curve. The highlighted curve is the case with the parameter setting of the third column of Table 1. It has resonances at the targeted frequencies with $\sim 600 \mathrm{Ohm}$ peak impedances. So with this approach the impedance peaks 
can be tuned to be nearly at the right bands with the right impedances and hence, no or minimal Cpar tuning is required.

An important note here is, that nearly half of the total required $\sim 14 \mathrm{pF}$ Cpar value is the Si4010 PA capacitance and the other half is realized by an SMD capacitor. As the Si4010 PA has lower $Q$ than the SMD with the $\sim 7 \mathrm{pF}$ PA capacitance setting the loss (Rres) value is determined more by the PA. The $2 \mathrm{~K}$ value of Rres here represents this. It is also shown in Table 1, that a small common inductance $(\operatorname{Lin}=1 \mathrm{nH})$ is allowed between the main and the small loop, so the antenna layout can be realized.

The conclusion is that by introducing a proper series Cser 2 cap in the small loop the dual band antenna structure can be tuned well with a much larger main loop size with a physically realizable small loop size and with a realizable antenna structure. The effect of the variation of the small loop size and Cser2 capacitance tuning is also shown in Fig. 16. The results of two other settings are shown: one with a smaller and another with a larger small loop size. Here the main loop size is constant and the series Cser 2 value is adjusted to have symmetrical peak shape.

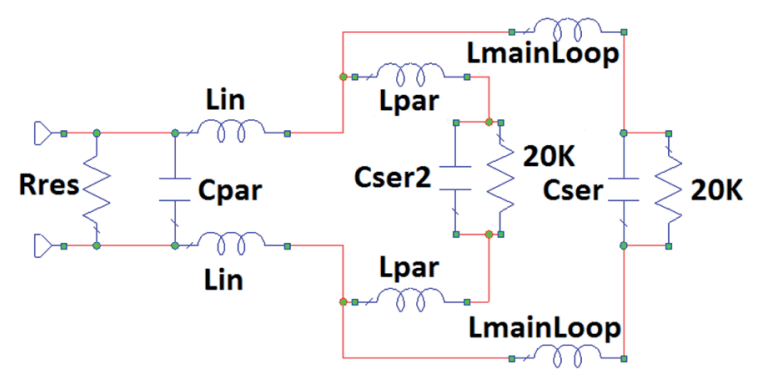

Fig. 15 Equivalent circuit of a dual band loop with a series cap introduced in the small loop (Cser2) as well.

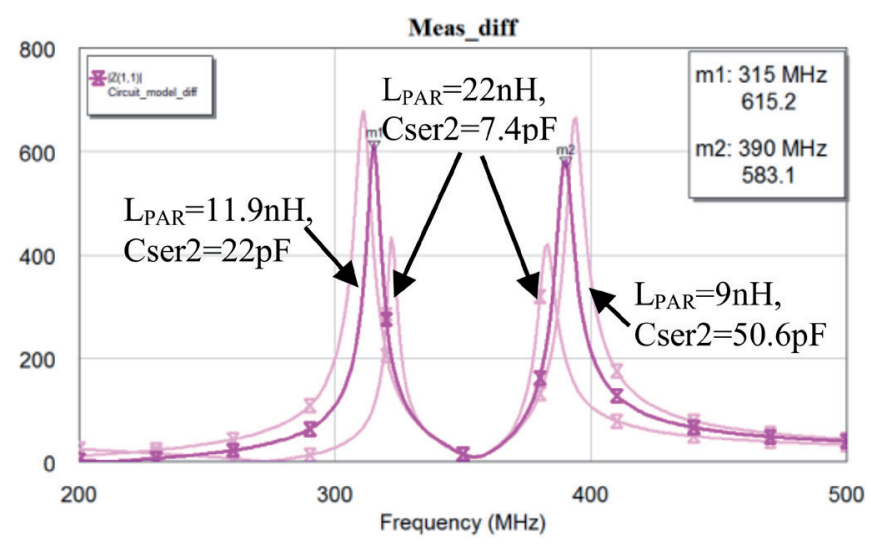

Fig. 16 Simulated impedance magnitude vs. frequency curves at different small loop sizes and Cser 2 capacitance values. The Cser 2 value is tuned to have a symmetrical two peak shape in all cases.

With larger small loop the offset and the impedance decreases. The opposite happen if the small loop size decreased. This clearly shows that by introducing the Cser 2 series cap in the small loop an additional degree of freedom appears in the design. With this additional tuning possibility one can adjust both the offset and peak impedance values according to the needs with the enlarged main loop size.

Applying the conclusions of the above investigations a dual band $315 / 390 \mathrm{MHz}$ loop antenna is designed and built (Fig. 17). The size of the main loop is $29 \times 38 \mathrm{~mm}$, the small loop dimensions are $5.5 \times 10 \mathrm{~mm}$. The simulated impedance magnitude curve are given in Fig. 18 with a fixed PA capacitance of $C$ par $=14 \mathrm{pF}$. The main loop series capacitance (Cser) in the simulation is $1.25 \mathrm{pF}$ while the small loop series capacitance (Cser2) is $21 \mathrm{pF}$.

There is possibility to design antennas for three or even more band operation with this method. For each band an additional loop with its own series caps has to be applied in the antenna. However, the design complexity increases rapidly. E.g. a three band antenna was successfully designed and measured as well, but not detailed in this paper.

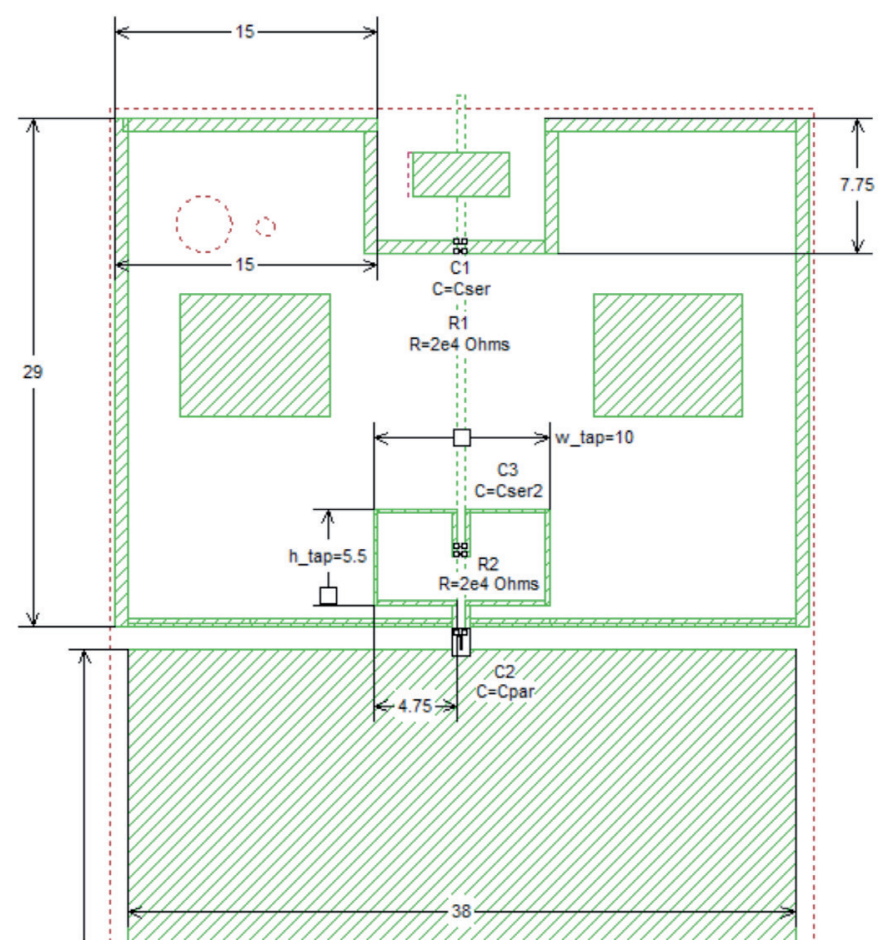

Fig. 17 Designed and built 315/390 MHz dual band loop antenna with 29x38 mm large main loop and with a Cpar of $14 \mathrm{pF}$.

\section{Impedance Measurement}

During the bench measurements a Cser cap of $1.3 \mathrm{pF}$ and Cser 2 cap of $22 \mathrm{pF}$ are used (1.25 pF and $21 \mathrm{pF}$ do not exists). Due to the slightly different $C$ ser and Cser 2 tuning is required in the bench. Also there are secondary effects (pcb cutting edge close to the antenna, battery on the gnd plane, nearby plastic etc.) which are cannot be taken into account in the $2.5 \mathrm{D}$ planar Sonnet simulations. Due to these, further decrease of Cpar value required. It should be emphasized again that a small change in the Cser capacitor and/or in the main loop parasitics 
require a large variation in the Cpar to compensate. Moreover, due to the high $Q$ structure the loop is sensitive to detuning.

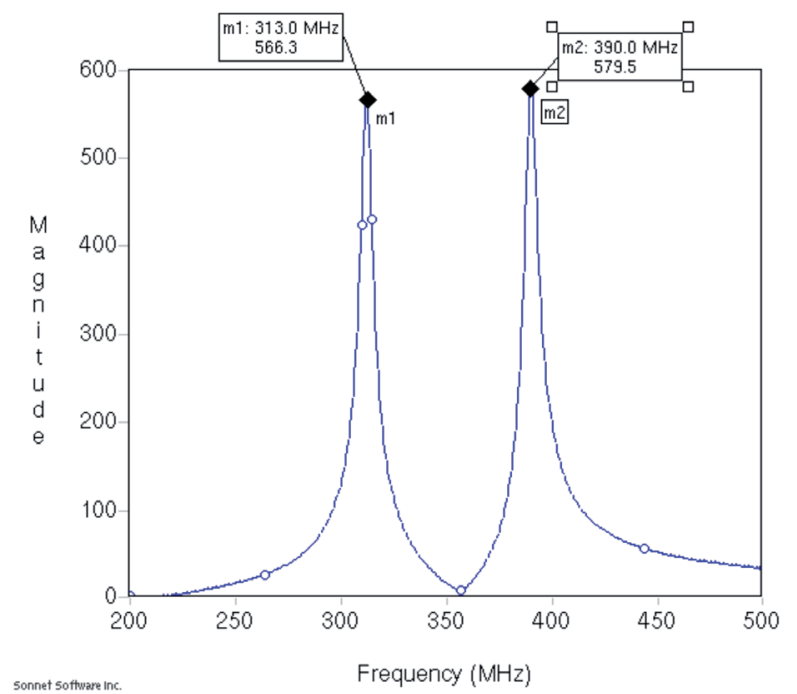

Fig. $18315 / 390 \mathrm{MHz}$ dual band loop antenna simulated impedance magnitude curve $($ Cpar $=14 \mathrm{pF}$, Cser $=1.25 \mathrm{pF}$, Cser $2=21 \mathrm{pF})$.

So the difference between the simulated and real tuned Cpar can be significant. The measured differential antenna impedance with 3.5 pF PA capacitance (Cpar) is shown in Fig. 19. The lower peak is at $315 \mathrm{MHz}$, but the upper resonance is at a higher frequency than the targeted $390 \mathrm{MHz}$. With Cpar tuning up to $\sim 9 \mathrm{pF}$ it can be tuned down to $390 \mathrm{MHz}$. Again this is done by the Si4010 automatic antenna tuning feature when the TX operating frequency is at $390 \mathrm{MHz}$.

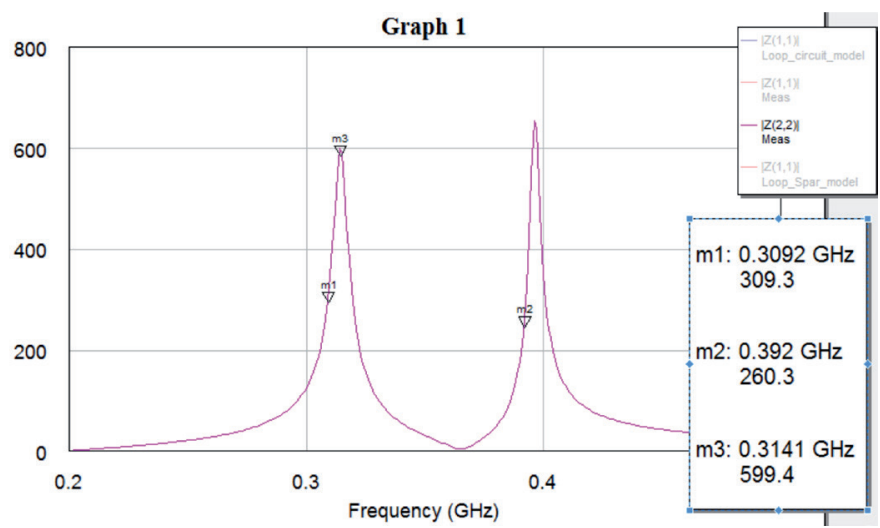

Fig. $19315 / 390 \mathrm{MHz}$ dual band loop antenna measured impedance magnitude curve $($ Par $=3.5 \mathrm{pF}$, Cser $=1.3 \mathrm{pF}$, Cser $2=22 \mathrm{pF})$.

\section{Radiation Simulations and Measurements}

The applied coordinate system for the radiation simulations and measurements are shown in Fig. 20. The simulated radiation characteristics at 310,315 and $390 \mathrm{MHz}$ are shown in Fig. 21. Here only the upper half hemisphere above the pcb is shown i.e. the $\theta$ runs between $-90^{\circ}$ and $90^{\circ}$. The $\varphi$ runs in $45^{\circ}$ steps so both the $\mathrm{ZX}\left(\varphi=0^{\circ}\right.$ or $\left.180^{\circ}\right)$ and $\mathrm{ZY}\left(\varphi=90^{\circ}\right.$ or $\left.-90^{\circ}\right)$ cut characteristic included as well.
Unfortunately, the 2.5D Sonnet simulator cannot simulate the radiation in the pcb plane well and thus, the $\theta=-90^{\circ}$ and $\theta=90^{\circ}$ results are not valid. The characteristic is symmetrical to the pcb plane i.e. has the same shape beneath the pcb.

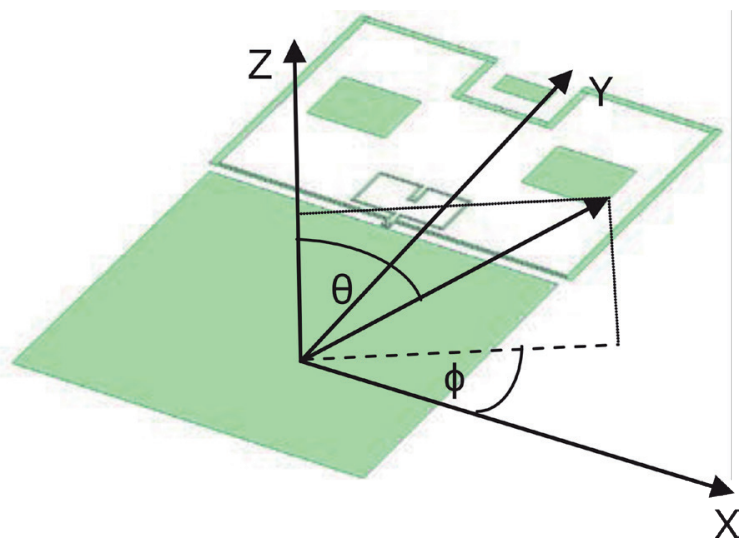

Fig. 20 Coordinate system used in the radiation simulations

As one can see the gain does not depend too much on the $\varphi$ at $390 \mathrm{MHz}$ so the pattern in the XY cut (parallel with the pcb) is fairly omnidirectional. The simulated gain maximum is $\sim-13.5$ $\mathrm{dBi}$ and $\sim-23 \mathrm{dBi}$ at $390 \mathrm{MHz}$ and $315 \mathrm{MHz}$ respectively.

The measured gain maximums of the antenna are $-8 \mathrm{dBi}$ and $-21 \mathrm{dBi}$ respectively. The measurement inaccuracy of the used test site is approx. $+-3 \mathrm{~dB}$ so the measured results are close to the simulated ones.
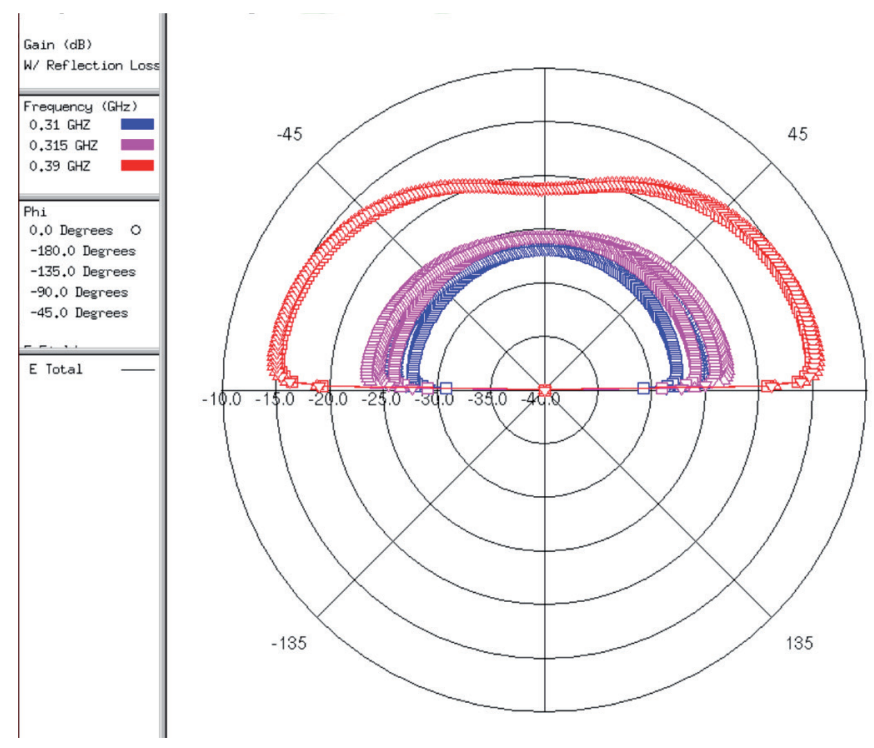

Fig. 21 Coordinate system used in the radiation simulations

\section{Conclusion}

A novel dual band loop antenna working in the UHF ISM bands is presented. With applying a proper series capacitance in the small loop practically realizable antenna structure is achievable. Tuning methods are shown to optimize the antenna performances at the two targeted frequencies. An antenna is designed, built and measured, which works well with the Si4010 RF IC at 315 and $390 \mathrm{MHz}$. 


\section{References}

[1] AbuTarboush, H. F., Nilavalan, R., Al-Raweshidy, H. S., Budimir, D. "Design of planar inverted-F antennas (PIFA) for multiband wireless applications." In: International Conference on Electromagnetics in Advanced Applications, 2009, ICEAA '09. pp. 78-81. 14-18 Sept. 2009. DOI: 10.1109/iceaa.2009.5297606

[2] Zólomy, A., Mernyei, F., Erdélyi, J., Pardoen, M., Tóth, G. "An Automatic Antenna Tuning for RF Transmitter IC Applying High Q Antenna." In: Radio Frequency Integrated Circuits (RFIC) Symposium, 2004. Digest of Papers. 2004 IEEE, pp. 501-504, 6-8 June 2004. DOI: $10.1109 /$ rfic.2004.1320666

[3] "Si4060/Si4460/61/67 Low-Power PA Matching." Silicon Laboratories Application Note: AN627 [Online] Available from: http://www.silabs. com/Support\%20Documents/TechnicalDocs/AN627.pdf [Accessed: 10th November 2014]
[4] Balanis, A. C. "Antenna Theory, Analysis and Design." $2^{\text {nd }}$ Ed., John Wiley and Sons, New York, 1997.

[5] Fujimoto, K., Henderson, A., Hirasawa, K., James, J. R. "Small Antennas." John Wiley and Sons, 1993.

[6] "Design of Printed Trace Differential Loop Antennas." Silicon Laboratories Application Note: AN639 [Online]. Available from: http:// www.silabs.com/Support\%20Documents/TechnicalDocs/AN639.pdf [Accessed: 27 July 2014]

[7] Si4010 datasheet, [Online]. Available from: https://www.silabs.com/ Support\%20Documents/TechnicalDocs/Si4010.pdf [Accessed: 23th February 2011]

[8] "Si4010 Calculator Spreadsheet Usage." Silicon Laboratories Application Note: AN547, [Online]. Available from: http://www.silabs.com/ Support\%20Documents/TechnicalDocs/AN547.pdf [Accessed: 18th January 2011]

[9] Sonnet high frequency 3D planar EM simulator, [Online]. Available from: http://www.sonnetsoftware.com/ [Accessed: since 1994] 